\title{
Impact of exercise on catechol-O-methyltransferase activity in depressive patients: A preliminary communication
}

\author{
Lara S. F. Carneiro', Maria Paula Mota1, Renato Sobral Monteiro-Junior ${ }^{2}$, \\ José Vasconcelos-Raposo ${ }^{3}$, Maria Augusta Vieira-Coelho ${ }^{4}$ \\ ${ }^{1}$ Research Centre in Sports Sciences, Health Sciences and Human Development, CIDESD, Vila Real, Portugal \\ ${ }^{2}$ Graduate Program of Health Sciences, State University of Montes Claros, Minas Gerais, Brazil \\ ${ }^{3}$ Research in Education and Community Intervention, RECl, Portugal \\ ${ }^{4}$ Psychiatry and Mental Health Clinic, Centro Hospitalar São João, Porto, Portugal
}

Catechol-O-methyltransferase (COMT) is a catabolic enzyme involved in the degradation of monoamines including the neurotransmitter dopamine (Williams, Owen, \& O'Donovan, 2007). In fact, a decreased level of endogenous dopaminergic neurotransmitter has been reported in depressive subjects (Pearson-Fuhrhop et al., 2014), as well as higher COMT activity in depressive patients in comparison to non-depressed subjects (Carneiro et al., 2016). Exercise has become increasingly accepted as an effective therapy in reducing depressive symptoms (Carneiro, Fonseca, Vieira-Coelho, Mota, \& Vasconcelos-Raposo, 2015). However, the neurobiological mechanisms underpinning this improvement remain poorly clarified (Schuch et al., 2015). The present study provides a key contribution to understand the paths by which exercise modulates the monoamine system. Indeed, the effect of exercise on COMT activity is unknown, and it remains to be explained if chronic exercise changes COMT activity.

This randomized control trial assesses the effects of chronic exercise on a soluble cytoplasmic isoform (S-COMT) activity in women with clinical depression.
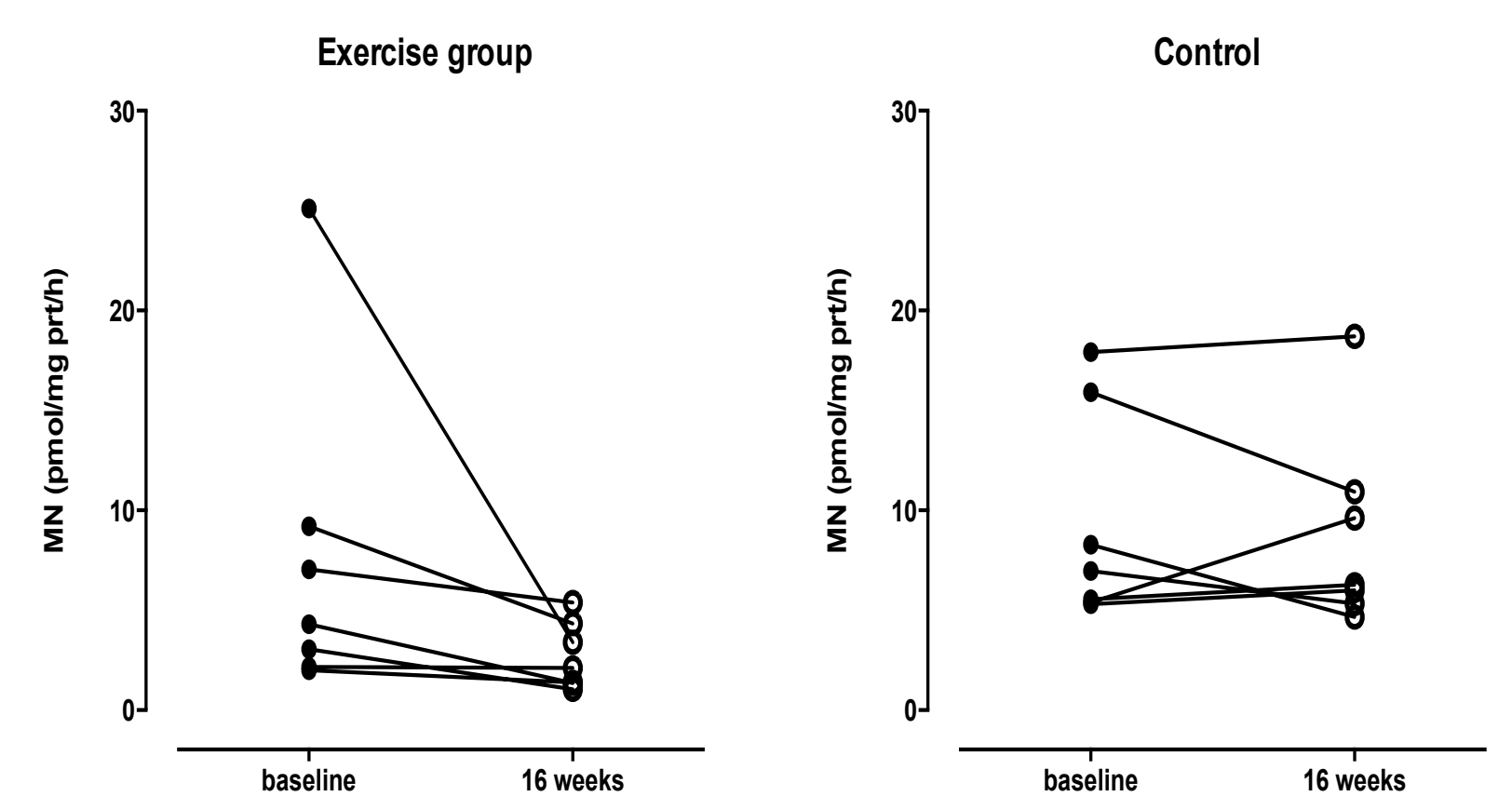

Figure 1 - Erythrocyte S-COMT activity (pmol/mg prot/h) in exercise group and controls at baseline and at the end of physical exercise program (W16). Exercise group- $p=0,0156$ within group by Wilcoxon test.

Controls $-p=0,8125$ within group by Wilcoxon test.
Fourteen women (aged: $51.4 \pm 10.5$ years) diagnosed with clinical depression (according to the International Classification of Diseases-10) were randomized to one of two groups: pharmacotherapy plus exercise $(n=7)$ or only pharmacotherapy $(n=7)$.

The aerobic exercise program comprised a $45-50 \mathrm{~min} / \mathrm{session}$, three times/week for 16 weeks. Erythrocyte soluble COMT has been evaluated before and after the exercise intervention. Exercise group in comparison to control group demonstrated a significant decrease $(p=0,02, r=-0,535)$ in S-COMT activity between baseline and after 16 weeks. Adding exercise to the usual treatment (pharmacotherapy) decreases significantly SCOMT activity levels of clinical depressed patients after 16 weeks. Our results provide evidence that exercise interferes with S-COMT activity, a molecular mechanism involved in depression.

\section{References}

Carneiro, L. S., Fonseca, A. M., Serrao, P., Mota, M. P., Vasconcelos-Raposo, J., \& Vieira-Coelho, M. A. (2016). Impact of physical exercise on catechol-O-methyltransferase activity in depressive patients: A preliminary communication. Journal of Affective Disorders 193:117-122.

Carneiro, L. S., Fonseca, A. M., Vieira-Coelho, M. A., Mota, M. P., \& Vasconcelos-Raposo, J. (2015). Effects of structured exercise and pharmacotherapy vs. pharmacotherapy for adults with depressive symptoms: A randomized clinical trial. Journal of Psychiatric Research 71: 48-55.

Pearson-Fuhrhop, K. M., Dunn, E. C., Mortero, S., Devan, W. J., Falcone, G. J., Lee, P., . . . Cramer, S. C. (2014). Dopamine genetic risk score predicts depressive symptoms in healthy adults and adults with depression. PLoS ONE 9(5): e93772.

Schuch, F. B., Deslandes, A. C., Stubbs, B., Gosmann, N. P., Silva, C. T., \& Fleck, M. P. (2015). Neurobiological effects of exercise on major depressive disorder: A systematic review. Neuroscience and Biobehavioral Reviews 61:1-11.

Williams, H. J., Owen, M. J., \& O'Donovan, M. C. (2007). Is COMT a susceptibility gene for schizophrenia? Schizophrenia Bulletin 33(3): 635-664. 